\title{
Large eddy simulation of hydrodynamic turbulence using renormalized viscosity
}

Sumit Vashishtha, ${ }^{1}$ Roshan Samuel, ${ }^{2, \text { a) }}$ Anando Gopal Chatterjee, ${ }^{1}$ Ravi Samtaney, ${ }^{3}$ and Mahendra K. Verma ${ }^{1}$

${ }^{1)}$ Department of Physics, Indian Institute of Technology, Kanpur 208016, India

${ }^{2)}$ Department of Mechanical Engineering, Indian Institute of Technology, Kanpur 208016, India

3) Physical Science and Engineering Division, King Abdullah University of Science and Technology, Thuwal 23955, Saudi Arabia

(Dated: 15 May 2019)

We employ renormalized viscosity to perform large eddy simulations (LES) of decaying homogeneous and isotropic turbulence in a cubical domain. We perform a direct numerical simulation (DNS) on $512^{3}$ and $256^{3}$ grids, and LES on $32^{3}, 64^{3}$, and $128^{3}$ grids with the same initial conditions in the resolved scales, for a flow with Taylor Reynolds number $\operatorname{Re}_{\lambda}=210$. We observe good agreement between LES and DNS results for the temporal evolution of turbulence kinetic energy $E_{u}(t)$, kinetic energy spectrum $E_{u}(k)$, and kinetic energy flux $\Pi_{u}(k)$. Also, the large-scale structures of the flow in LES are similar to those in DNS. These results establish the suitability of our renormalized viscosity scheme for LES.

PACS numbers: 64.60.ae, 47.11.-j, 47.11.Kb

Keywords: Renormalized viscosity, large eddy simulations, turbulence

\section{INTRODUCTION}

Turbulence is one of the most challenging problems in classical physics. Direct numerical simulations (DNS) at extreme Reynolds numbers (Re) remain infeasible even on the fastest contemporary supercomputers. This is because of the rapid increase in the required grid size, $\operatorname{Re}^{9 / 4}$, for large Re. Therefore an alternative strategy, large eddy simulation (LES), becomes one of the most efficient techniques for simulating turbulent flows.

In LES, only the large scales of turbulent flows are simulated, and the unresolved scales are appropriately modeled $^{1-3}$. In turbulence, Fourier modes corresponding to different length scales interact with each other. According to Kolmogorov's theory of turbulence ${ }^{4,5}$, the nonlinear interactions in a turbulent flow yield a constant energy flux from large scales to intermediate scales, and then to small scales. For fluid flow at length scales belonging to the inertial range, the energy flux $\Pi_{u}$ is equal to the energy dissipation rate. Various turbulence models have been designed to describe this energy flux in both the inertial and dissipative ranges ${ }^{6}$. Also, the spectral eddy viscosity ${ }^{7}$ at length scale $l$ is proportional to $\Pi_{u}^{1 / 3} l^{4 / 3}$. Physically, the momentum diffuses with above enhanced viscosity. This idea is exploited in LES.

Smagorinsky $^{8}$ constructed the first subgrid-scale (SGS) model in which the effects of small scales are accounted for using the eddy viscosity, defined as

$$
\nu_{\text {Smag }}=\left(C_{s} \Delta\right)^{2} \sqrt{2 \bar{S}_{i j} \bar{S}_{i j}}
$$

where $\bar{S}_{i j}$ is the stress tensor at the resolved scales, $\Delta$ is the smallest grid scale, and $C_{s}$ is a constant whose

\footnotetext{
a)Electronic mail: roshanj@iitk.ac.in
}

value typically lies between 0.1 and 0.2 . Deardorff ${ }^{9}$ was the first to implement this model in a numerical simulation. We expect $C_{s}$ to be different at different flow regimes of inhomogeneous and anisotropic turbulence. For such flows, Germano et al. ${ }^{10}$ proposed a dynamic Smagorinsky model that utilises two filters - a grid filter, and a test filter. The constant $C_{s}$ is determined using the difference of the two stress tensors computed for these filter widths. Also see Lilly ${ }^{11}$, Meneveau and Katz $^{1}$, Lesieur and Metais ${ }^{2}$, and Sagaut ${ }^{3}$ for detailed discussions. Newer methods to compute $C_{s}$, like scaledependent Lagrangian models, are in use to solve complex atmospheric flows like wind-turbine wakes ${ }^{12}$.

Kraichnan ${ }^{7}$ used two point closures to compute the spectral eddy viscosity, $\nu_{\text {tot }}\left(k, k_{c}\right)$, where $k_{c}$ is the wavenumber cutoff based on the grid size of the LES. For a sharp spectral cutoff, a cusp develops in $\nu_{\text {tot }}\left(k, k_{c}\right)$ near $k=k_{c}{ }^{7,13}$. Dantinne et al. ${ }^{14}$ addressed this issue for pseudo-spectral simulations. Chollet and Lesieur ${ }^{15}$ employed the eddy viscosity model by Kraichnan ${ }^{7}$ to study spectral properties of homogeneous turbulence. Métais and Lesieur ${ }^{16}$, and Lesieur and Metais ${ }^{2}$ devised schemes to implement the same in real space. The resulting expressions are related to the velocity structure functions. In Sarghini's LES model ${ }^{17}$, scale-similarity of the flow structures above and below the cutoff is exploited to capture backscatter, which is defined as the energy transfer from subgrid scales to large scales ${ }^{18}$.

Prinz et al. ${ }^{19}$ employed a new mixed subgrid-scale model based on velocity increment tensor and eddyviscosity $^{20}$ to perform LES of wall-bounded hydrodynamic and magnetohydrodynamic flows. More recently, Dupuy et al. ${ }^{21}$ investigated a wide variety of LES models and compared their performances for wall-bounded flows. There has also been an analytical attempt to combine LES equations with Reynolds-Averaged Navier 
Stokes (RANS) equations ${ }^{22}$. Menon et al. ${ }^{23}$ performed both DNS and LES of forced and decaying isotropic turbulence to compare various subgrid models for LES. They found that dynamic one-equation models like the scalesimilarity model perform better than Smagorinsky models. We specifically focus on decaying, homogeneous, isotropic turbulence for testing the proposed LES model in this paper. The study of this form of turbulence is of practical importance as it has been shown to manifest drag-reduction-type phenomena, specifically in the presence of additives ${ }^{24,25}$.

Several other LES models ${ }^{26,27}$ model the subgrid stress using the velocity field rather than the resolved stress tensor, $\bar{S}_{i j}$. In the works of Misra and Pulin ${ }^{27}$ and Cheng et al. ${ }^{28}$, the subgrid viscous stresses are computed using the properties of stretched vortices whose orientations are determined by the resolved velocity field. This model has also been extended to compressible decaying isotropic turbulence in Kosović et al. ${ }^{29}$. There are variations of the above models, as well as attempts to fine-tune the LES models for complex flows involving confined and complex geometries, boundary layers, etc. However, we do not detail such models because they are beyond the scope of this paper.

A less popular LES model is based on the renormalized viscosity, which is computed using renormalization group (RG) analysis ${ }^{30-34}$. RG analysis helps us understand systems with multiple scales, turbulence being one such problem. Therefore, this field-theoretic approach has been used to formulate closure schemes for homogeneous isotropic turbulence ${ }^{35}$. In Wilson's Fourier-space RG scheme, Fourier space is divided into many shells, and the nonlinear interactions among various shells are computed using first-order perturbation theory that yields a scale-dependent viscosity called renormalized viscosity. Here we state the formula for this renormalized viscosity $\nu_{\text {ren }}$ :

$$
\nu_{\text {ren }}(k)=K_{\mathrm{Ko}}^{1 / 2} \Pi_{u}^{1 / 3} k^{-4 / 3} \nu_{*},
$$

where $k$ is the wavenumber, $\nu_{*}$ is a constant, and $K_{\text {Ko }}$ is Kolmogorov's constant. Using RG computation, McComb and Watt ${ }^{36}$ found that $\nu_{*}=0.50$ and $K_{\mathrm{Ko}}=1.62$. Verma $^{37}$ computed the above quantities and found $\nu_{*}=$ 0.38 and $K_{\mathrm{Ko}}=1.6$. Note that the above formula has been derived from first principles (from the Navier-Stokes equation) for homogeneous and isotropic turbulence under certain assumptions ${ }^{30-34}$.

This renormalized viscosity of Eq. (2) is very similar to that employed in the Smagorinsky model (see Eq. (1)). For a subgrid cutoff of $\Delta$ (in real space), the cutoff wavenumber is $k_{c}=\pi / \Delta$. Hence, the renormalized viscosity to be employed for LES would be

$$
\nu_{\mathrm{ren}}\left(k_{c}\right)=K_{\mathrm{Ko}}^{1 / 2} \Pi_{u}^{1 / 3} k_{c}^{-4 / 3} \nu_{*} .
$$

Note that due to the presence of $\Pi_{u}$ in the above equation, $\nu_{\text {ren }}$ is expected to vary with time. This temporal variation is seen in Fig. 1 (c) and is discussed in Sec. IV.
Moreover, we observe inverse cascade or negative $\Pi_{u}$ in $2 \mathrm{D}$ turbulence. This can make the subgrid-scale energy transfer sign-indefinite, and this is one of the key problems in the modelling of LES $^{38}$. Note that our proposed LES scheme is valid for 3D flows only, and its generalization to $2 \mathrm{D}$ requires major revision.

We can easily demonstrate an equivalence between Eq. (1) and Eq. (3). By converting Equation (1) to Fourier space, we obtain

$$
\begin{aligned}
\nu_{\text {Smag }} & =\left(C_{s} \frac{\pi}{k_{c}}\right)^{2}\left[\int_{0}^{k_{c}} 2 k^{2} E_{u}(k) d k\right]^{1 / 2} \\
& \approx\left(C_{s} \frac{\pi}{k_{c}}\right)^{2}\left[\frac{3}{2} K_{\mathrm{Ko}} \Pi_{u}^{2 / 3} k_{c}^{4 / 3}\right]^{1 / 2} \\
& =C_{s}^{2} \pi^{2}\left[\frac{3}{2} K_{\mathrm{Ko}}\right]^{1 / 2} \Pi_{u}{ }^{1 / 3} k_{c}^{-4 / 3}
\end{aligned}
$$

where $E_{u}(k)$ is the energy spectrum, which is taken to be Kolmogorov's spectrum as an approximation. Now a comparison of Eq. (1) and Eq. (3) yields

$$
C_{s}=\frac{\nu_{*}^{1 / 2}}{\pi}\left(\frac{2}{3}\right)^{1 / 4} .
$$

For $\nu_{*}=0.38$, we obtain $C_{s}=0.177$, which lies within the range of values employed in LES. The above analysis demonstrates that the RG technique may be used to compute the undetermined constants of the subgrid stress terms in LES.

Most of the RG computations, however, are for homogeneous and isotropic turbulence ${ }^{30-34}$. Hence the constants (e.g $\nu_{*}, K_{\mathrm{Ko}}$ ) computed using these calculations are not suitable for anisotropic and inhomogeneous flows. Hence, extensive work is required for realistic estimation of the parameters from the first-principle calculations. There have been limited attempts to generalise it to anisotropic scenarios ${ }^{39}$.

However, before expanding the scope of RG computations to complex flows, it is important to validate the isotropic eddy viscosity model of Eq. (3) using numerical simulations. For the same, one can compare the results of RG-based LES with those from DNS. Verma and Kumar ${ }^{40}$ performed one such analysis for decaying hydrodynamic turbulence in a periodic box. They performed a DNS on a $128^{3}$ grid and a LES on a $64^{3}$ grid with the same parameters, and showed that the evolution of total energy, as well as the energy spectrum, in DNS and LES are in good agreement with each other. In this paper, we compare results of DNS on two larger grids of $512^{3}$ and $256^{3}$ with LES results on coarser-grids of $32^{3}, 64^{3}$, and $128^{3}$. We perform extensive validation tests for such LES, and show that the energy evolution, energy spectra, energy fluxes, and instantaneous large scale structures of LES and DNS match quite well. We also highlight the emergence of bottleneck effect in LES.

The outline of the paper is as follows: In Sec. II, we describe the governing equations and the renormalized 
viscosity model used in LES. Computational methodologies are discussed in Sec. III. The results obtained from LES and DNS are described in Sec. IV. Finally, we summarize our results in Sec. V.

\section{LES FORMULATIONS USING RENORMALIZED PARAMETERS}

In this section, we present the formalism of LES using renormalized parameters. The incompressible NavierStokes equations in real space are

$$
\begin{aligned}
\frac{\partial \mathbf{u}}{\partial t}+\mathbf{u} . \nabla \mathbf{u} & =-\nabla p+\nu_{0} \nabla^{2} \mathbf{u}, \\
\nabla \cdot \mathbf{u} & =0,
\end{aligned}
$$

where $\mathbf{u}$ is the velocity vector, $\mathrm{p}$ is the pressure, and $\nu_{0}$ is the kinematic viscosity. When solving the above equations on a coarser grid, the subgrid fluctuations of the flow variables are filtered out. Therefore, the filtered form of the equations are

$$
\begin{aligned}
\frac{\partial \overline{\mathbf{u}}}{\partial t}+\overline{\mathbf{u}} \cdot \nabla \overline{\mathbf{u}} & =-\nabla \bar{p}+\nu_{0} \nabla^{2} \overline{\mathbf{u}}+\nabla \cdot \tau^{\Delta}, \\
\nabla \cdot \overline{\mathbf{u}} & =0,
\end{aligned}
$$

where $\nabla \cdot \tau^{\Delta}$ is the subgrid stress term that arises from filtering. This term must be modeled in order to accurately represent the physics of the flow. The representation of various scales in a flow is more convenient in Fourier space. Hence, most of the RG analysis of turbulent flows have been performed in Fourier space. We write the corresponding incompressible fluid-flow equations in Fourier space as

$$
\begin{gathered}
\left(\frac{d}{d t}+\nu_{0} k^{2}\right) \hat{u}_{j}(\mathbf{k}, t)= \\
-\frac{i}{2} P_{j l m}(\mathbf{k}) \sum_{\mathbf{p}, \mathbf{q}} \delta_{\mathbf{k}, \mathbf{p}+\mathbf{q}} \hat{u}_{l}(\mathbf{p}) \hat{u}_{m}(\mathbf{q}), \\
k_{i} \hat{u}_{i}(\mathbf{k})=0
\end{gathered}
$$

where

$$
\begin{aligned}
P_{j l m}(\mathbf{k}) & =k_{l} P_{j m}(\mathbf{k})+k_{m} P_{j l}(\mathbf{k}), \\
P_{j l}(\mathbf{k}) & =\delta_{j l}-\frac{k_{j} k_{l}}{k^{2}} .
\end{aligned}
$$

The right hand side of Eq. (10) represents the triadic interactions among the wavenumbers $\mathbf{k}, \mathbf{p}$ and $\mathbf{q}$ such that $\mathbf{k}=\mathbf{p}+\mathbf{q}$. Note that the definition of Fourier transform used here is:

$$
\mathbf{u}(\mathbf{x}, t)=\sum_{\mathbf{k}} \hat{\mathbf{u}}(\mathbf{k}, t) e^{i \mathbf{k} \cdot \mathbf{x}} .
$$

In RG scheme, the Fourier modes of wavenumber shells are truncated iteratively ${ }^{30-34}$. This leads to the elimination of some of these triadic interactions. RG computation takes into account these interactions, and puts these effects into an enhanced viscosity. It has been shown that the total effective viscosity at wavenumber $k_{c}$ is

$$
\nu_{\text {tot }}=\nu_{0}+\nu_{\mathrm{ren}}\left(k_{c}\right)=\nu_{0}+K_{\mathrm{Ko}}^{1 / 2} \Pi_{u}^{1 / 3} k_{c}^{-4 / 3} \nu_{*} .
$$

For details of RG procedure, please refer to Yakhot and Orszag $^{30}$, Zhou et al. ${ }^{33}$, McComb ${ }^{41}$ and Verma ${ }^{42}$.

The LES scheme based on renormalized viscosity makes use of Eq. (15). We employ a sharp spectral filter at the cutoff wavenumber $k_{c}$ :

$$
\hat{\overline{\mathbf{u}}}(\mathbf{k}, t)=H\left(k_{c}-k\right) \hat{\mathbf{u}}(\mathbf{k}, t),
$$

where $H$ represents the Heaviside function. Hence, the real space velocity is

$$
\overline{\mathbf{u}}(\mathbf{x}, t)=\sum_{\mathbf{k}} e^{i \mathbf{k} \cdot \mathbf{x}} \hat{\overline{\mathbf{u}}}(\mathbf{k}, t)=\sum_{|\mathbf{k}|<\left|\mathbf{k}_{\mathbf{c}}\right|} e^{i \mathbf{k} \cdot \mathbf{x}} \hat{\mathbf{u}}(\mathbf{k}, t) .
$$

With this, the equations for the resolved Fourier modes are

$$
\begin{gathered}
\left(\frac{d}{d t}+\nu_{\mathrm{tot}} k^{2}\right) \hat{\bar{u}}_{j}(\mathbf{k})= \\
-\frac{i}{2} k_{j} P_{j l m}(\mathbf{k}) \sum_{\mathbf{p}, \mathbf{q} \leq \mathbf{k}_{\mathbf{c}}} \delta_{\mathbf{k}, \mathbf{p}+\mathbf{q}} H\left(k_{c}-k\right) \hat{u}_{l}(\mathbf{q}) \hat{u}_{m}(\mathbf{p}) \\
\mathbf{k} \cdot \hat{\mathbf{u}}(\mathbf{k})=0 .
\end{gathered}
$$

The total viscosity, $\nu_{\text {tot }}$, in Eq. (18) is computed using Eq. (15), where $\nu_{*}=0.38$, and $K_{\mathrm{Ko}}=1.6$. Note that renormalized viscosity $\nu_{\text {ren }}(k)$ is computed at the cutoff wavenumber $k_{c}$, which is assumed to lie in the inertial range.

The computation of $\nu_{\text {tot }}$ for LES requires the Kolmogorov's flux $\Pi_{u}\left(k_{0}\right)$, where $k_{0}$ is in the inertial range. In our simulations, we compute $\Pi_{u}\left(k_{0}\right)$ using the modeto-mode formula of Verma ${ }^{37}$ and Dar et al. ${ }^{43}$ :

$$
\Pi_{u}\left(k_{0}\right)=\sum_{k \geq k_{0}} \sum_{p<k_{0}} \delta_{k, p+q} \operatorname{Im}[\mathbf{k} \cdot \mathbf{u}(\mathbf{q})]\left[\mathbf{u}^{*}(\mathbf{k}) \cdot \mathbf{u}(\mathbf{p})\right] .
$$

Regarding the choice of $k_{c}$ in an $N^{3}$ periodic box of length $2 \pi$, we take $k_{c}=N / 3$ due to dealising employed in our DNS and LES. Under the $2 / 3$ rule of dealiasing, the Fourier modes $|\mathbf{k}|>N / 3$ are set to zero (Note that $\left.k_{\max }=N / 2\right)$. Hence, the nonzero Fourier modes are $k_{i}=[-N / 3: N / 3]$, where $i=x, y, z$. Therefore, for our DNS and LES, the effective $k_{c}=N / 3$. We remark that this scheme is superior to that employed by Verma and Kumar ${ }^{40}$, who take $k_{c}=N / 2$ and the viscous dissipation rate as an estimate for the energy flux $\Pi_{u}$. Consequently, the agreement between LES and DNS in the present model is much better than that obtained by Verma and Kumar ${ }^{40}$.

In the following section, we discuss different computational methodologies of our simulations. 


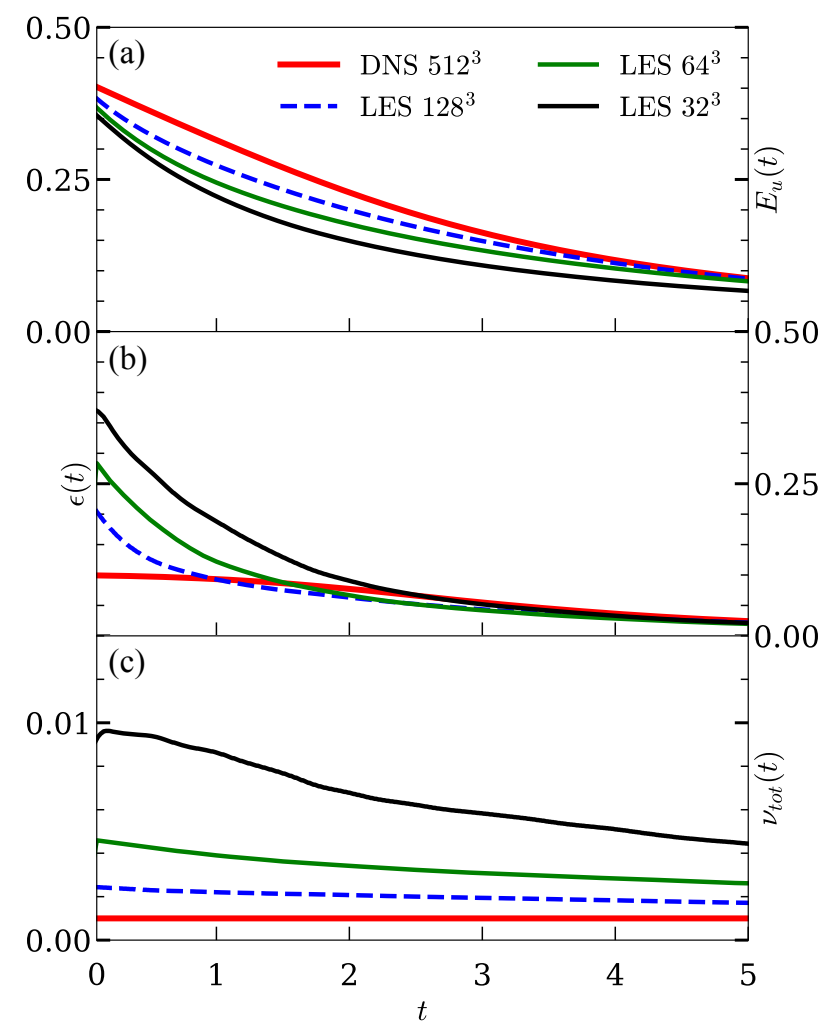

FIG. 1. (a) Temporal evolution of total kinetic energy $E_{u}(t)=$ $u^{2} / 2$ for DNS on a $512^{3}$ grid and for LES on $32^{3}, 64^{3}$, and $128^{3}$ grids. The evolution of $E_{u}(t)$ is similar for the DNS and LES runs. (b) Temporal evolution of total dissipation $\epsilon(t)$ for the DNS and three LES. (c) Temporal evolution of total viscosity $\nu_{\text {tot }}=\nu_{0}+\nu_{\text {ren }}$ for the three LES. For all LES, $\nu_{\text {tot }}$ decreases with time, and it reaches $\nu_{0}$ asymptotically. $\nu_{\text {tot }}$ is largest for $32^{3}$ LES due to $k_{c}^{-4 / 3}$ factor of Eq. (15), while $\nu_{\text {tot }}=\nu_{0}$ for DNS.

\section{SIMULATION DETAILS}

We solve Eqs. $(10,11)$ in our DNS, and Eq. $(18,19)$ in our LES using pseudo-spectral method. We use the code Tarang ${ }^{44,45}$ for the same. We perform DNS computations on $512^{3}$ and $256^{3}$ grids, and LES computations on $32^{3}, 64^{3}$ and $128^{3}$ grids. For these simulations, we use a periodic cube of size $2 \pi \times 2 \pi \times 2 \pi$, hence the wavenumber components are integers. In our simulations, we employ the fourth-order Runge-Kutta method for timemarching, and the Courant-Friedrichs-Lewy (CFL) condition for determining the time-step $\Delta t$. Furthermore, the $2 / 3$ rule $^{46}$ is used for dealiasing. We take $\nu_{0}=10^{-3}$ for DNS and three LES.

First, we perform a forced DNS on $512^{3}$ grid with $\nu_{0}=$ $10^{-3}$, and evolve the flow to a steady state. We use the final state of this DNS as an initial condition for the decaying DNS on both $512^{3}$ and $256^{3}$ grids, as well as for the decaying LES runs. Note that under this scheme, the Fourier modes of the LES at the resolved scales are
TABLE I. Details of Reynolds numbers, Courant numbers, time-steps and number of iterations to reach up till $t=2$ for the $512^{3}$ DNS run and the $128^{3}$ LES run.

\begin{tabular}{lcccc}
\hline \hline Case & Re & $C_{\mathrm{CFL}}$ & $\mathrm{dt}$ & Iterations \\
\hline $512^{3} \mathrm{DNS}$ & 953 & 0.05 & $3 \times 10^{-5}$ & 66,667 \\
$128^{3} \mathrm{LES}$ & 938 & 0.05 & $5 \times 10^{-3}$ & 400 \\
\hline \hline
\end{tabular}

exactly same as those in DNS. The Reynolds number based on Taylor's micro-scale $\operatorname{Re}_{\lambda} \approx 210$ for the flow field corresponding to the initial condition. For the LES, we take $k_{c}=N / 3$ and viscosity as given in Eq. (15). For the DNS, $\nu_{\text {tot }}=\nu_{0}$. The decaying simulations have been carried out with non-dimensional time unit of $L / U$, where $L$ and $U$ are the large length scale and velocity scale respectively.

In the next section, where we compare the results of LES and DNS, we have chosen a relatively short timeinterval of 2.0 non-dimensionalized units. However, a sufficiently large number of time-steps has elapsed in this interval. In Table I, we show the time-steps and efficiency of the LES model. For simulations of decaying turbulence, it is difficult to expect similarities between two runs to persist for long time as the flow becomes viscous. Finally, the Reynolds numbers are moderately high at the start of the simulations. Moreover, to be safe, the Courant number is chosen to be much smaller than unity. As a result, the time-steps used in our simulations are rather small, and can be increased under a less strict value of Courant number.

\section{COMPARISON OF DNS AND LES RESULTS}

In this section, we compare the DNS and LES results on the evolution of the total energy, total dissipation rate, the energy spectrum and flux, as well as the real space structures. First we start with the evolution of total energy $E_{u}(t)$ and dissipation rate $\epsilon(t)$ for DNS and LES, which are defined as

$$
\begin{aligned}
E_{u, \mathrm{DNS}}(t) & =\frac{1}{2} \sum_{k}|\hat{\mathbf{u}}(\mathbf{k}, t)|^{2}, \\
E_{u, \mathrm{LES}}(t) & =\frac{1}{2} \sum_{k}|\hat{\mathbf{u}}(\mathbf{k}, t)|^{2}, \\
\epsilon_{\mathrm{DNS}}(t) & =\sum_{k} 2 \nu_{0} k^{2} E_{u}(k), \\
\epsilon_{\mathrm{LES}}(t) & =\sum_{k} 2 \nu_{\mathrm{tot}} k^{2} \bar{E}_{u}(k) \\
& =\sum_{k} 2\left(\nu_{0}+\nu_{\mathrm{ren}}\left(k_{c}\right)\right) k^{2} \bar{E}_{u}(k),
\end{aligned}
$$

where $\hat{\mathbf{u}}(\mathbf{k})$ represents the Fourier components of the resolved velocity used for LES, while $\hat{\mathbf{u}}(\mathbf{k})$ is the full velocity field used for DNS. $\bar{E}_{u}(k)$ is the total energy of the resolved Fourier modes in LES.

In Fig. 1(a), we exhibit the temporal evolution of total turbulent kinetic energy $E_{u}(t)$. We observe that 

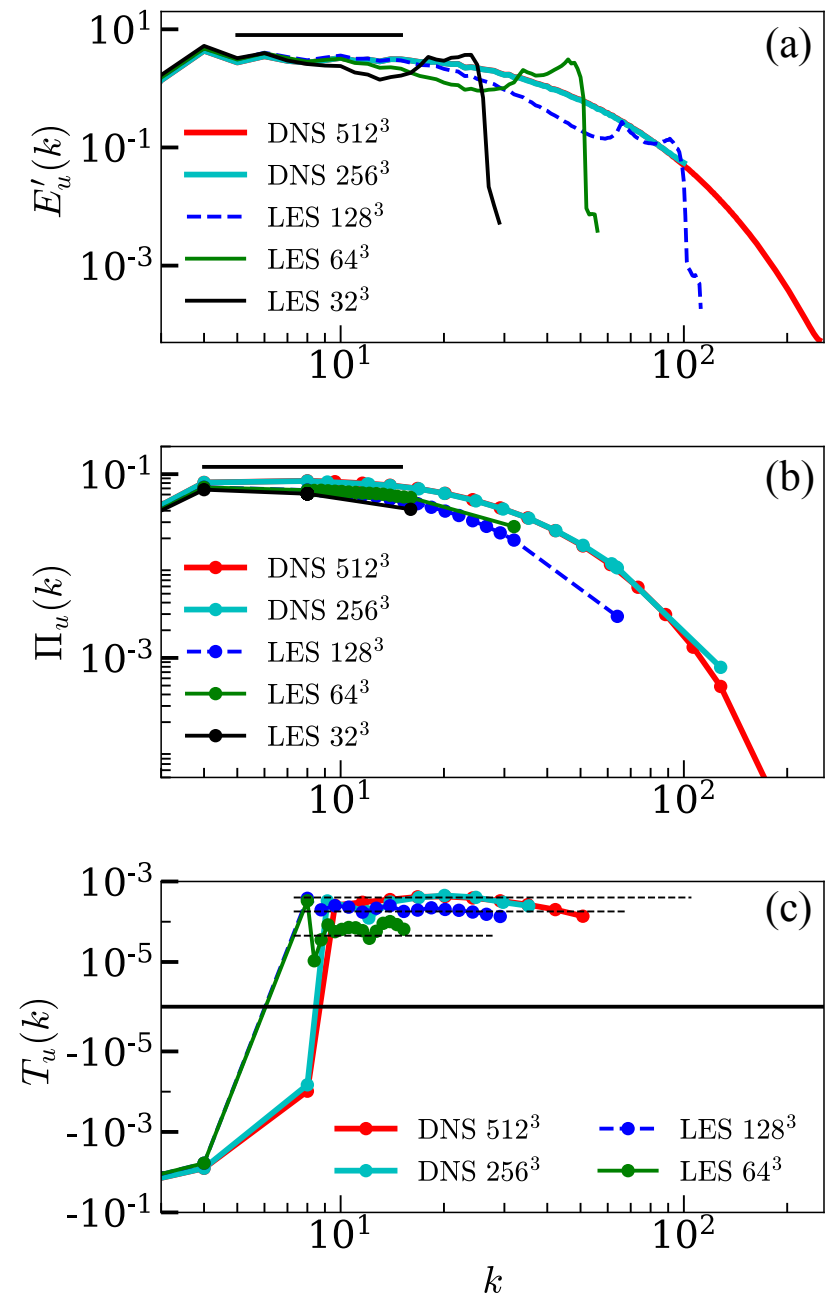

FIG. 2. (a) The normalized kinetic energy spectra $E_{u}^{\prime}(k)=E_{u}(k) k^{5 / 3} \Pi_{u}^{-2 / 3}$ for DNS and LES. In the inertial range, $E_{u}^{\prime}(k)$ is approximately equal for all the runs. The LES runs exhibit bumps in $E_{u}^{\prime}(k)$ due to the bottleneck effect. (b) The kinetic energy fluxes $\Pi_{u}(k)$ for DNS and LES. In the inertial range, $\Pi_{u}(k)$ are approximately constant for all the runs. (c) The transfer spectra $T_{u}(k)=-d \Pi_{u}(k) / d k$ for DNS and LES. The kinetic energy transfer is reduced for the coarse grained LES. This strengthens the justification that the truncation of high wavenumber modes reduces energy transfer. The spectra and fluxes are plotted at $t=2$.

the evolution of $E_{u}(t)$ for all the runs are quite similar. Note, however, that the initial energy of the lowerresolution LES is smaller than that of DNS and the higher-resolution LES. The lower initial energy observed in the LES runs is because of the fewer number of modes present in these runs.

In Fig. 1(b) we plot $\epsilon(t)$ vs. $t$ for different simulations. Note that at early stages, $\epsilon(t)$ is larger for LES with lower resolutions; this feature can be explained as follows. Ac-

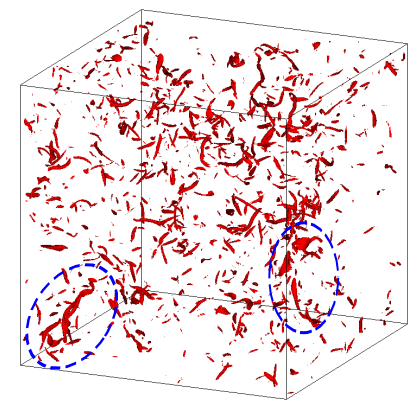

(a)

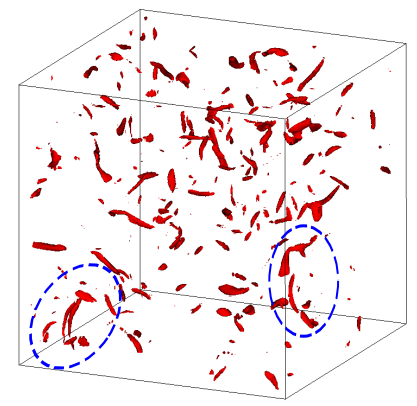

(b)
FIG. 3. Isosurfaces of $|\omega(\mathbf{x})|$ for (a) DNS $512^{3}$ and (b) LES $128^{3}$ at $t=2$. Clearly, LES with $1 / 4$ th grid resolution in each direction captures the large-scale structures of DNS. The blue dashed outlines in each figure highlight the similarity between the vortical structures in the two decaying simulations.

cording to Eq. (24), $\epsilon_{\mathrm{LES}}(t)$ is affected by two factors: (a) the number of modes over which summation is performed, and (b) $\nu_{r}\left(k_{c}\right)$, which is proportional to $k_{c}^{-4 / 3}$. Note that with decreasing grid resolutions, $\epsilon(t)$ decreases due to (a), but it increases due to (b). We observe that $\epsilon(t)$ is affected more by (b) than by (a). This is the reason for the observed increase in dissipation rate with decrease of grid resolution. Asymptotically (at large $t$ ), $\epsilon(t)$ for all the runs reach the same value because $\nu_{\text {tot }} \rightarrow \nu_{0}$ as $t \rightarrow \infty$.

In Fig. 1(c), we illustrate the total viscosity $\nu_{\text {tot }}=\nu_{0}+\nu_{\text {ren }}$ as a function of time. Among all the LES, the one with the smallest grid $\left(32^{3}\right)$ has the largest $\nu_{\text {tot }}$ because $k_{c}$ is smallest for this run. Also, $\nu_{\text {tot }}=\nu_{0}$ for DNS. As time progresses, the total energy decreases for all the runs and the flow becomes viscous. Asymptotically, $\nu_{\text {tot }} \rightarrow \nu_{0}$.

Figure 2 exhibits the normalized kinetic energy spectrum, $E_{u}^{\prime}(k)=E_{u}(k) k^{5 / 3} \Pi_{u}^{2 / 3}$ (top), the kinetic energy flux, $\Pi_{u}(k)$ (middle), and the kinetic energy transfer specrum, $T_{u}(k)=-d \Pi_{u}(k) / d k$ (bottom), at $t=2$. In the inertial range, $E_{u}^{\prime}(k)$ computed using DNS and LES are approximately same. In addition, as shown in Fig. 2(b), the energy flux $\Pi_{u}(k)$ for all the runs is also constant in the inertial range. This constant flux is also seen in different turbulence models ${ }^{6}$. This indicates that our LES model can capture the physics of the flow in the inertial range quite well. Note however that $\Pi_{u}(k)$ decreases as the resolution of LES is lowered. This is because the truncated large wavenumber modes eliminate some of the nonlinear triads, thus decreasing the nonlinear coupling and the energy flux. This is also demonstrated in Fig. 2(c), where the kinetic energy transfer spectra are shown for the DNS and LES runs. We observe that the energy transfer is diminished for the coarse-grained LES, as highlighted by the thin dashed lines. 
TABLE II. Unresolved kinetic energy $E_{\text {ur }}$ in non-dimensional units, and expressed as percentage fraction of the total kinetic energy, for LES simulations at $t=1,1.5$ and 2 units.

\begin{tabular}{lcccccc}
\hline \hline \multirow{2}{*}{ Case } & \multicolumn{2}{c}{$t=1.0$} & \multicolumn{2}{c}{$t=1.5$} & \multicolumn{2}{c}{$t=2.0$} \\
\cline { 2 - 7 } & $E_{\text {ur }}\left(\times 10^{-2}\right)$ & $\% E_{\text {ur }}$ & $E_{\text {ur }}\left(\times 10^{-2}\right)$ & $\% E_{\text {ur }}$ & $E_{\text {ur }}\left(\times 10^{-2}\right)$ & $\% E_{\text {ur }}$ \\
\hline $128^{3}$ LES & 4.78 & 12.8 & 4.79 & 14.9 & 4.13 & 15.0 \\
$64^{3}$ LES & 5.33 & 14.3 & 6.25 & 19.4 & 5.68 & 20.7 \\
$32^{3}$ LES & 7.22 & 19.4 & 8.67 & 26.9 & 8.46 & 30.8 \\
\hline \hline
\end{tabular}

Note that the energy spectrum is plotted over a span of uniformly spaced wavenumbers. However, the kinetic energy flux is plotted for specific shell radii. We highlight this in Fig. 2(b) with filled circle markers. This is because flux calculation is a computationally expensive step and we choose the shell radii judiciously to minimize the time taken. For instance, in the $128^{3}$ LES, we had chosen the spherical shell radii as $k=0,2,4,8,8.77$, 9.62 , and so on, because our focus is on the inertial range. Therefore, the $T_{u}(k)$ 's at small wavenumbers are not appropriately resolved. Moreover, due to this difference in the wavenumbers chosen for spectrum and flux calculations, the plots extend over slightly different ranges in the three panels of Fig. 2.

In Fig. 2(a), we observe bumps in $E_{u}^{\prime}(k)$ near the end of inertial regime. The height of the bump increases as the grid resolution is decreased. This feature can be attributed to the bottleneck effect ${ }^{47,48}$ wherein the suppression of energy at the small scales makes the energy transfer less efficient; this leads to an accumulation of energy near the cutoff wavenumber $k_{c}$. Note that in LES, the total viscosity (Fig. 1(c)) increases with lower grid resolutions. Thus, in LES, the bottleneck effect gets enhanced as the grid resolution decreases.

In Table II, we list the unresolved kinetic energy $\left(E_{\mathrm{ur}}\right)$ in non-dimensional units for the LES runs at three different times, $t=1.0,1.5$ and 2.0. $E_{\mathrm{ur}}$ is obtained by subtracting the area under the LES spectrum (shown for $t=2$ in Fig 2(a)) from that under the DNS spectrum. We also express this energy as percentage fraction of the total kinetic energy in the corresponding DNS run. We observe that $E_{\mathrm{ur}}$ is decreasing between $t=1.5$ to 2.0 for all the LES runs. However, this decrease is because of the overall decay of global kinetic energy as the turbulence decays. This fact is reflected by the percentage $E_{\mathrm{ur}}$ which is increasing within the same time interval. Moreover, the $E_{\mathrm{ur}}$ is higher for LES with coarser grid because more of the high wavenumber modes get truncated as the $k_{c}$ reduces.

After the above diagnostics in spectral space, we compare the DNS and LES results in real space. For the same, we present the isosurfaces of the magnitude of vorticity, $|\omega(\mathbf{x})|$, at a given time. In Fig. 3 we compare these plots of $|\omega(\mathbf{x})|$ for DNS and LES at $t=2$. Note that our LES with $1 / 4$ th the resolution in each direction as compared to DNS captures the structures of DNS quite satisfactorily. Many large-scale structures in the LES are

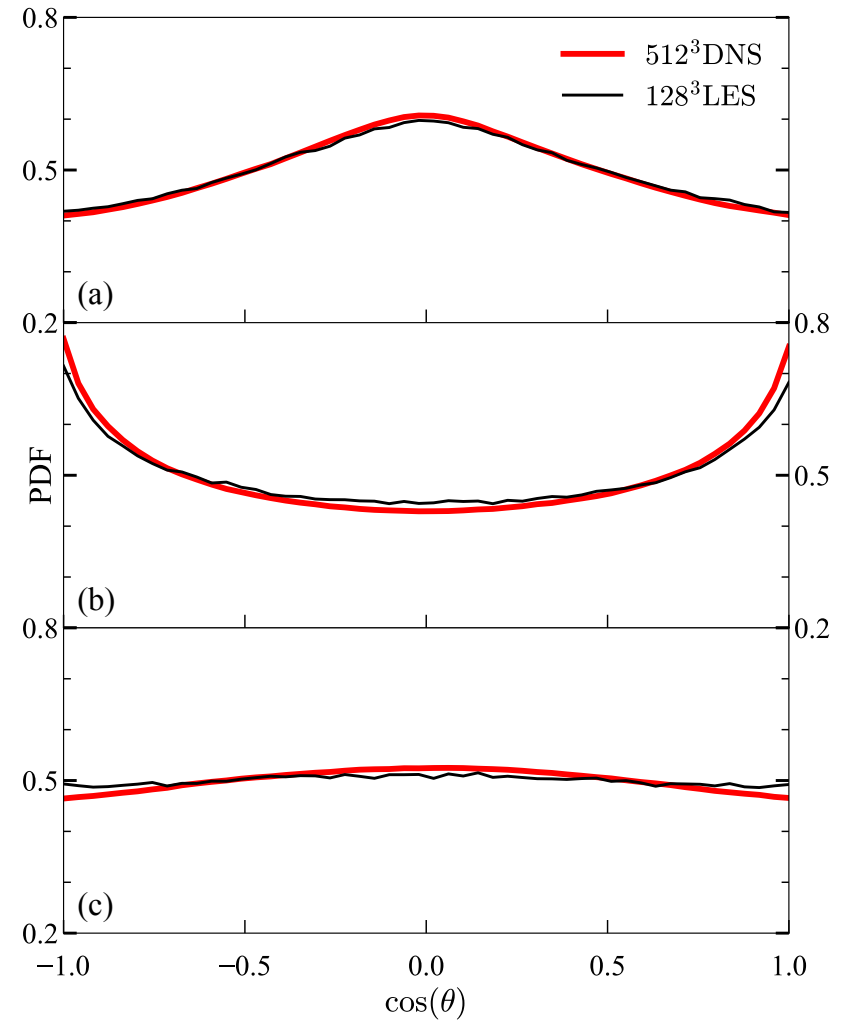

FIG. 4. Probability Density Functions of the angles between the vorticity vector and the three principal directions of the strain-rate tensor computed at $t=2.0$. The three angles are those corresponding to the smallest (a), intermediate (b), and largest (c) eigenvalues. The PDFs are computed from the same data as plotted in Fig. 3.

observed to correspond very well with those seen in the DNS, as highlighted with dashed regions in the figure.

Additionally, we demonstrate the accuracy of our LES model through the alignment of vorticity vectors with the three principal directions of the strain-rate tensor $\left(e_{i j}\right)$. In Fig. 4, we plot the probability density functions of the cosines of the three angles between vorticity and the eigenvectors of $e_{i j}$, where $e_{i j}$ is given by

$$
e_{i j}=\frac{1}{2}\left(\frac{\partial u_{i}}{\partial x_{j}}+\frac{\partial u_{j}}{\partial x_{i}}\right)
$$

The plots in Fig. 4 (a), (b), and (c) correspond to the 

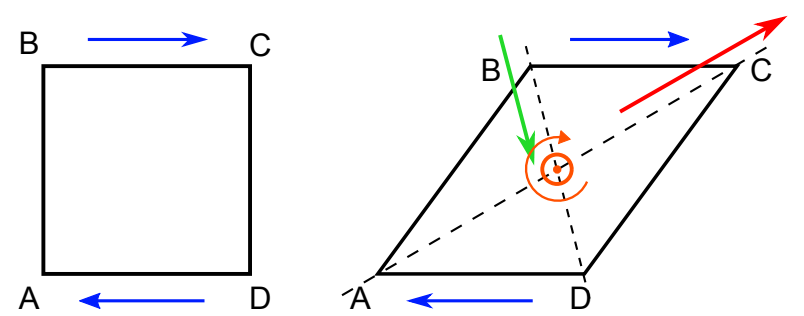

FIG. 5. Vorticity can be expected to align perpendicular to the two principal directions of extremal eigenvalues of the strain-rate tensor $\left(e_{i j}\right)$. This can explain the PDFs seen in Fig. 4.

smallest, intermediate, and largest eigenvalues of $e_{i j}$ respectively. The PDFs have been plotted for both $512^{3}$ DNS (thick red line) and $128^{3}$ LES (thin black line), and we observe good agreement between the PDFs for the 2 smallest eigenvalues. We observe that the vorticity vector tends to align preferentially with the eigenvector corresponding to the intermediate eigenvalue, and perpendicular to the eigenvectors of the smallest and largest eigenvalues. Physically, the extremal eigenvalues correspond to the directions of compression and stretching of the fluid parcels. The sketch shown in Fig. 5 demonstrates how the vorticity tends to align along the intermediate eigenvector. The above results provide validation for our LES scheme.

The LES model used here mainly takes local interactions into account. However 3D turbulence also involves non-local interactions which can be captured using higher-order structure functions. In Linkmann et al. ${ }^{49}$, a comparison of the correlation functions in LES and DNS was performed. These correlation matrices appear in the evolution equations for structure functions. We plan to perform such a comparison in the future. We conclude in the next section.

\section{CONCLUSIONS}

In the present work, we employ renormalized viscosity to perform LES of decaying turbulence. We compare the LES and DNS results and show that the LES can capture the evolution of total energy and total dissipation rate, as well as the energy spectrum and flux with much coarser grids than that of DNS. In real space, the large-scale structures of LES are quite similar to those of DNS. We use these results to validate our LES model based on renormalized viscosity.

We however remark that the present model is not suitable for anisotropic and inhomogeneous turbulence, as seen in flows close to walls. Yakhot et al. ${ }^{50}$ employed RG-based ideas to simulate anisotropic flows, and Chasnov $^{13}$ employed EDQNM stochastic model to capture backscatter. In future, we will generalise our LES model to take such effects into account.

We perform LES in a periodic box, thus excluding the effects of boundary layer. Boundary layers significantly alter the flow due to strong velocity gradients. We need to generalize our renormalized viscosity model to such flows. One approach to do this would be to employ local kinetic energy fluxes, because they could potentially capture the variability of dissipation rates. We may compute such fluxes using the third-order velocity structure functions locally ${ }^{51}$. We plan such generalizations in future.

In summary, renormalization-group based LES offers interesting possibilities that needs further explorations.

\section{ACKNOWLEDGMENTS}

We thank Manohar Sharma, Syed Fahad Anwer, and Shashwat Bhattacharya for useful discussions. The simulations were performed on the HPC system, funded by DST, and Chaos cluster of IIT Kanpur, India. Some of our computations were also performed on Shaheen II at KAUst Supercomputing Laboratory, Saudi Arabia, under the project $\mathrm{k} 1052$. This work was supported by the research grant PLANEX/PHY/2015239 from Indian Space Research Organisation (ISRO), India.

\section{REFERENCES}

${ }^{1}$ Charles Meneveau and Joseph Katz. Scale-invariance and turbulence models for large-eddy simulation. Annual Review of Fluid Mechanics, 32(1):1-32, 2000.

${ }^{2} \mathrm{M}$. Lesieur and O. Metais. New trends in large-eddy simulations of turbulence. Annu. Rev. Fluid Mech., 28(1):45-82, 1996.

${ }^{3}$ Pierre Sagaut. Large eddy simulation for incompressible flows: an introduction. Springer-Varlag, Berlin, 2006.

${ }^{4}$ Andrey Nikolaevich Kolmogorov. Dissipation of Energy in Locally Isotropic Turbulence. Dokl Acad Nauk SSSR, 32(1):16-18, 1941.

${ }^{5}$ Andrey Nikolaevich Kolmogorov. The local structure of turbulence in incompressible viscous fluid for very large Reynolds numbers. Dokl Acad Nauk SSSR, 30(4):301-305, 1941.

${ }^{6}$ M. K. Verma, A. Kumar, P. Kumar, S. Barman, A. G. Chatterjee, R. Samtaney, and R. A. Stepanov. Energy spectra and fluxes in dissipation range of turbulent and laminar flows. Fluid Dynamics, 53(6):862-873, 2018.

${ }^{7}$ Robert H. Kraichnan. Eddy viscosity in two and three dimensions. J. Atmos. Sci., 33:1521-1536, January 1976.

${ }^{8}$ Joseph Smagorinsky. General circulation experiments with the primitive equations: I. the basic experiment. Monthly weather review, 91(3):99-164, 1963.

${ }^{9}$ James W Deardorff. A numerical study of three-dimensional turbulent channel flow at large Reynolds numbers. J. Fluid Mech., 41:453-480, 1970 .

${ }^{10}$ Massimo Germano, Ugo Piomelli, Parviz Moin, and William H. Cabot. A dynamic subgrid-scale eddy viscosity model. Phys. Fluids A, 3(7):1760-1765, July 1991.

${ }^{11}$ D K Lilly. A proposed modification of the Germano subgrid-scale closure method. Phys. Fluids A, 4:633-635, March 1992.

${ }^{12}$ Mahdi Abkar and Fernando Porté-Agel. Influence of atmospheric stability on wind-turbine wakes: A large-eddy simulation study. Physics of Fluids, 27(3):035104, 2015.

${ }^{13}$ Jeffrey R Chasnov. Simulation of the kolmogorov inertial subrange using an improved subgrid model. Phys. Fluids A, 3(1): 188-200, 1991. 
${ }^{14}$ G Dantinne, Hervé Jeanmart, GS Winckelmans, Vincent Legat, and Daniele Carati. Hyperviscosity and vorticity-based models for subgrid scale modeling. Appl. Sci. Res., 59(4):409-420, 1997.

${ }^{15}$ Jean-Pierre Chollet and Marcel Lesieur. Parameterization of small scales of three-dimensional isotropic turbulence utilizing spectral closures. J. Atmos. Sci., 38(12):2747-2757, 1981.

${ }^{16}$ Olivier Métais and Marcel Lesieur. Spectral large-eddy simulation of isotropic and stably stratified turbulence. J. Fluid Mech., 239:157-194, 1992

${ }^{17} \mathrm{~F}$ Sarghini, U Piomelli, and E Balaras. Scale-similar models for large-eddy simulations. Phys. Fluids, 11(6):1596-1607, 1999.

${ }^{18}$ Ugo Piomelli, William H Cabot, Parviz Moin, and Sangsan Lee. Subgrid-scale backscatter in turbulent and transitional flows. Physics of Fluids A: Fluid Dynamics, 3(7):1766-1771, 1991.

${ }^{19}$ Sebastian Prinz, Thomas Boeck, and Jörg Schumacher. Large eddy simulation of hydrodynamic and magnetohydrodynamic channel flows with a collocated finite-volume scheme and improved subgrid-scale modeling. European Journal of Mechanics - B/Fluids, 72:189 - 198, 2018.

${ }^{20}$ Andrea Cimarelli and Elisabetta De Angelis. The physics of energy transfer toward improved subgrid-scale models. Physics of Fluids, 26(5):055103, 2014.

${ }^{21}$ D. Dupuy, A. Toutant, and F. Bataille. A posteriori tests of subgrid-scale models in an isothermal turbulent channel flow. Physics of Fluids, 31(4):045105, 2019.

${ }^{22}$ Stefan Heinz. The large eddy simulation capability of reynoldsaveraged navier-stokes equations: Analytical results. Physics of Fluids, 31(2):021702, 2019.

${ }^{23}$ S. Menon, P. K. Yeung, and W. W. Kim. Effect of subgrid models on the computed interscale energy transfer in isotropic turbulence. Computers \& Fluids, 25(2):165 - 180, 1996.

${ }^{24}$ Prasad Perlekar, Dhrubaditya Mitra, and R. Pandit. Manifestations of drag reduction by polymer additives in decaying, homogeneous, isotropic turbulence. Phys. Rev. Lett., 97(26):264501, January 2006.

${ }^{25}$ Prasad Perlekar, Dhrubaditya Mitra, and R. Pandit. Direct numerical simulations of statistically steady, homogeneous, isotropic fluid turbulence with polymer additives. Phys. Rev. E, 82(6):066313, December 2010.

${ }^{26} \mathrm{~J}$ Andrzej Domaradzki and Eileen M Saiki. A subgrid-scale model based on the estimation of unresolved scales of turbulence. Phys. Fluids, 9(7):2148-2164, 1997.

${ }^{27}$ Ashish Misra and Dale I Pullin. A vortex-based subgrid stress model for large-eddy simulation. Phys. Fluids, 9(8):2443-2454, 1997.

${ }^{28}$ W. Cheng, Dale I Pullin, and Ravi Samtaney. Large-eddy simulation of separation and reattachment of a flat plate turbulent boundary layer. J. Fluid Mech., 785:78-108, November 2015.

${ }^{29}$ Branko Kosović, Dale I. Pullin, and Ravi Samtaney. Subgridscale modeling for large-eddy simulations of compressible turbulence. Physics of Fluids, 14(4):1511-1522, 2002.

${ }^{30}$ Victor Yakhot and Steven A. Orszag. Renormalization group analysis of turbulence. I. Basic theory. J. Sci. Comput., 1(1): 3-51, March 1986.

${ }^{31} \mathrm{~W}$ David McComb. The physics of fluid turbulence. Clarendon Press, Oxford, 1990.

${ }^{32} \mathrm{~W}$ David McComb. Homogeneous, Isotropic Turbulence: Phenomenology, Renormalization and Statistical Closures. Oxford University Press, 2014.
${ }^{33}$ Ye Zhou, George Vahala, and Murshed Hossain. Renormalization-group theory for the eddy viscosity in subgrid modeling. Phys. Rev. A, 37(7):2590-2598, April 1988.

${ }^{34}$ Ye Zhou. Renormalization group theory for fluid and plasma turbulence. Phys. Rep., 488(1):1-49, February 2010.

${ }^{35}$ Arjun Berera, Matthew Salewski, and W. D. McComb. Eulerian field-theoretic closure formalisms for fluid turbulence. Phys. Rev. $E$, 87(1):013007, January 2013.

${ }^{36} \mathrm{~W}$. D. McComb and AG Watt. Two-field theory of incompressible-fluid turbulence. Phys. Rev. A, 46(8):4797-4812, October 1992.

${ }^{37}$ Mahendra K. Verma. Statistical theory of magnetohydrodynamic turbulence: recent results. Phys. Rep., 401(5):229-380, November 2004.

${ }^{38}$ Gregory L Eyink and Hussein Aluie. Localness of energy cascade in hydrodynamic turbulence. I. Smooth coarse graining. Phys. Fluids, 21:115107, 2009.

${ }^{39} \mathrm{D}$ Carati and L Brenig. Renormalization-group method for anisotropic turbulent transport. Phys. Rev. A, 40(9):5193, 1989.

${ }^{40}$ Mahendra K. Verma and Shishir Kumar. Large-eddy simulations of fluid and magnetohydrodynamic turbulence using renormalized parameters. Pramana-J. Phys., 63(3):553-561, January 2004.

${ }^{41} \mathrm{~W}$ David McComb. Renormalization Methods: A Guide For Beginners. Oxford University Press, Oxford, 2004.

${ }^{42}$ M. K. Verma. Introduction to Statistical Theory of Fluid Turbulence. eprint arXiv:nlin/0510069, October 2005.

${ }^{43}$ Gaurav Dar, Mahendra K. Verma, and Vinayak Eswaran. Energy transfer in two-dimensional magnetohydrodynamic turbulence: formalism and numerical results. Physica D, 157(3):207225, January 2001.

${ }^{44}$ Mahendra K. Verma, Anando G. Chatterjee, Rakesh K. Yadav, Supriyo Paul, Mani Chandra, and Ravi Samtaney. Benchmarking and scaling studies of pseudospectral code Tarang for turbulence simulations. Pramana-J. Phys., 81(4):617-629, September 2013.

${ }^{45}$ Anando G Chatterjee, Mahendra K. Verma, Abhishek Kumar, Ravi Samtaney, Bilel Hadri, and Rooh Khurram. Scaling of a Fast Fourier Transform and a pseudo-spectral fluid solver up to 196608 cores. J. Parallel Distrib. Comput., 113:77-91, March 2018.

${ }^{46}$ Claudio Canuto, M Yousuff Hussaini, Alfio Quarteroni, and Thomas A Zang. Spectral Methods in Fluid Dynamics. SpringerVerlag, Berlin Heidelberg, 1988.

${ }^{47}$ Gregory Falkovich. Bottleneck phenomenon in developed turbulence. Phys. Fluids, 6(4):1411-1414, 1994.

${ }^{48}$ Mahendra K. Verma and Diego A. Donzis. Energy transfer and bottleneck effect in turbulence. J. Phys. A: Math. Theor., 40 (16):4401-4412, March 2007.

${ }^{49}$ Moritz Linkmann, Michele Buzzicotti, and Luca Biferale. Multiscale properties of large eddy simulations: correlations between resolved-scale velocity-field increments and subgrid-scale quantities. Journal of Turbulence, 19(6):493-527, 2018.

${ }^{50} \mathrm{~A}$. Yakhot, Steven A. Orszag, Victor Yakhot, and M. Israeli. Renormalization group formulation of large-eddy simulations. $J$. Sci. Comput., 4(2):139-158, June 1989.

${ }^{51}$ Uriel Frisch. Turbulence: The Legacy of A. N. Kolmogorov. Cambridge University Press, Cambridge, 1995. 